TITLE: ACTINIDE SPECIATION BY PHOTOTHERMAL SPECTROSCOPIES: INSTRUMENTATION DEVELOPMLNT

AUTHOR(S): J. M. Berg, C. D. Tait, D. E. Morris, and W. H. Woodruff

\author{
SUBMITTED TO: Materials Research Society \\ Boston, MA \\ Nov. $26-30,1990$
}

\title{
DISCLAIMER
}

This report was prepared as an account of work sponsored by an agency of the United States Government. Neither the United States Government nor any agency thereof, nor any of their employees, makes any warranty, express or implied, or assumes any legal liability or responsibility for the accuracy, completeness, or usefulness of any information, apparatus, product, or process disclosed, or represents that its use would not infringe privately owned rights. Reference herein to any specific commercial product, process, or service by trade name, trademark, manufacturer, or otherwise does not necessarily constitute or imply its endorsement, recommendation, or favoring by the United States Government or any agency thereof. The views and opinions of authors expressed herein do not necessarily state or reflect those of the United States Government or any agency thereof.

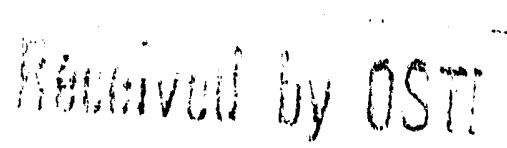

NOV 051990

Ste: :

By acceptarice of this article, the publisher recognizes that the US Government retains a nonexclusive, ruyalty-free license to publish or reproduce the published form of inis contribution. or to allow others to do so, for U.S Government purposes

The LoS Alamios National Laboratory requests that the publisher identify this arficle as work performed under the auspices of the $U$ S Department of Energy 


\section{ACTINIDE SPECLATION BY PHOTOTHERMAL SPECTROSCOPIES: INSTRUMENTATION DEVELOPMENT}

J. M. Berg, C. D. Tait, D. E. Morris and W. H. Woodruff; Isotope and Nuclear Chemistry Division; Los Alamos National Laboratory; Los Alamos, NM 87545

\section{ABSTRACT}

Photoacoustic spectroscopy using pulsed laser excitation is being developed by a number of research groups as one of the most promising methods for studying speciation of actinides in solution at environmentally relevant concentrations. We present details of a number of hardware and software techniques we have implemented $\omega$ improve the sensitivity of the method. Our approach is based on more extensive waveform analysis. While most signal processing techniques extract the analytical signal from only a small portion of the acoustic waveform produced in the detector by an absorption event, we describe two methods that use more of the waveform. Other methods of minimizing noise sources using both hardware and software are also described.

\section{INTRODUCTION}

Spectroscopic sndies of actinide complexes in aqueous solution promise to be useful aids for sorting out the speciation issues relevant to nuclear waste storage and waste site remediation. The electronic transitions of the actinide metal center can be quite sensitive to the ligand environment. Thus it is possible that spectroscopic signatures can be used to characterize uniquely particular species or families of species.

One of the significant barriers to the use of spectroscopic methods for studying actinide speciation is that typical solubilities of the metals under environmentally relevant conditions are in the sub-micromolar range, resulting in optical densities below $10^{-5}$ for reasonable sample path lengths. Conventional, absorption-based spectroscopies are not sensitive at this level. Thus it is important to develop spectroscopic methods that have the requisite sensitivity. A number of groups have employed photoacoustic spectroscopy' (PAS) or photothermal lensing spectroscopy ${ }^{2}$ (PTL) because they are sensitive enough and applicable to non-fluorescing systems like most actinides in solution are expected to be. ${ }^{3}$

We have constructed a laser photoacoustic spectroscopy system for actinide speciation studies which incorporates a number of modifications in hardware and signal processing techniques compared to versions described in published papers. One drawback of the prevailing signal processing techniques is that they use only a small amount of the total acoustic energy reaching the detector to produce spectum. We have implemented two techniques to capture more of the information-containing signal with the goal of increasing the sensitivity of the method. In the first approach a digitizing oscilloscope is used to acquire, digitally rectify and sum the waveform. The second approach uses an analog processing module to accomplish the equivalent result in real time for each laser shot. Both techniques use much more of the detected acoustic energy to produce a spectrum than the more common gated integrator technique. The advantages and disadvantages of these 
approaches relative to tach other and to the more widely used gated integrator signal processing are discussed. Recent efforts using thermal lensing detection are also discussed briefly.

We have also implemented a number of real-time statistical tests into our data acquisition software to minimize some of the more common noise and systematic error sources in the PAS method. These aid in reducing error due to one-time events which are not connected with absorption by either the analyte or the solvent, such as interactions with stray particulate matter in the solution.

Our primary test case has been $\mathrm{Nd}^{3+}$ at mictomolar concentrations. This ion has sharp $(f, f)$ absorption bands in the same wavelength regions as actinides of interest, and does not present the difficulties associated with handling radionuclides, allowing us to evaluate techniques much more rapidly. We have also obtained spectra of Pu(IV) rolutions under well controlled solution conditions in the $1-10 \mu \mathrm{M}$ range.

\section{EXPERMENTAL}

Photoacoustic optical spectroscopy as applied here utilizes the detection of acoustic energy released through nonradiative decay of optically-pumped excited states to measure electronic absorption profiles of metal complexes at low concentrations. Typically, a ceramic piezoelectric crystal is used as a detector. Placed in acoustic contact with the sample, it converts pressure fluctuations due to absorption and subsequent heat-releasing non-radiative decay into voltage fluctuations. These can then be amplified and measured in some way to produce an indication of the degree of absorption of a monochromatic laser pulse by the sample with which it is in acoustic contact. In our system, with $5 \mathrm{~ns}$ pulsed laser excitation and a relatively thick piezoelectric crystal $(3.2 \mathrm{~mm})$, the raw photoacoustic signal appears as a complicated ac voltage waveform with dominant frequencies around $100 \mathrm{kHz}$ and an envelope decay time of $\sim 100 \mu \mathrm{sec}$ after pulsed excitation.

Most published descriptions of techniques for extracting relative absorption data from this waveform involve using a gated integrasor to capture and integrate part of a single excursion of this waveform at early times when it is the most intense. Figure la shows a typical waveform at low signal levels and the gate over which one might integrate to measure the signal. The integrated intensity of this region of the waveform versus excitation warelength gives the PAS spectrum. One drawback of this signal processing technique is that it looks only at a small fraction of the acoustic waveform generated by photoexcitation (less than 5\% in our system.) A second potential drawback is that selection of the optimum time window for gated detection is important. There is some theoretical basis for using narrower gating to achieve improved signal-to-noise through better exclusion of background signal resulting from electrostriction effects. 4 However, this requires prior knowledge of noise sources and their effects on the waveform to select the optimum window. This information is frequently not available. Thus, it may be hetter to capture large portions of the waveform to maximize detected signal. 

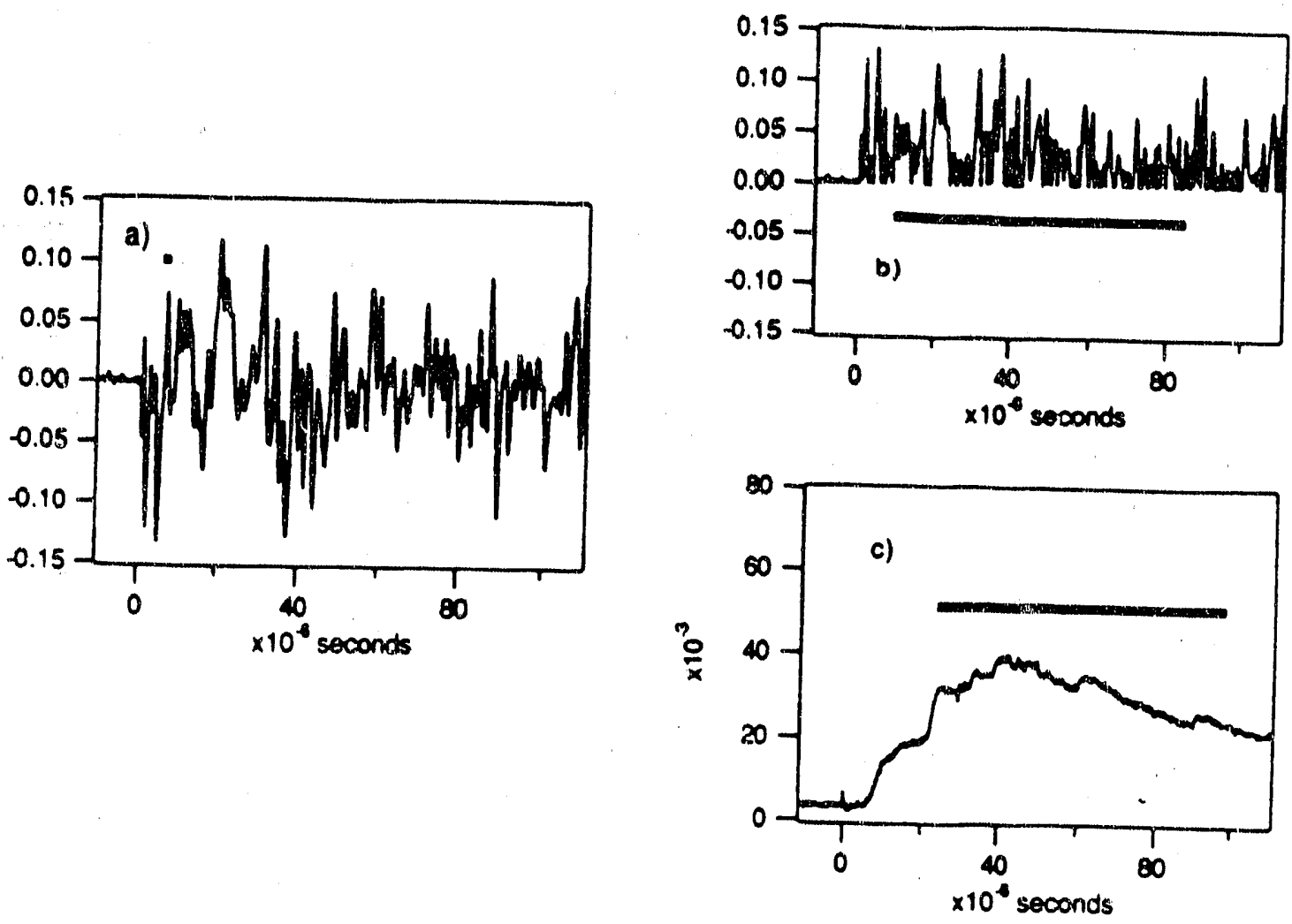

Figure 1. Waveform processing techniques: a) average of 64 amplified voltage waveforms from a $10 \mu \mathrm{M} \mathrm{Nd}^{3+}$ in $0.1 \mathrm{~N} \mathrm{HCl}$ solution, with boxcar gate placement indicated by a bar above the spectrum; b) digitized waveform after rectification, with integration gate indicated by a bar, c) waveform after processing by analog rectification ci:cuit, with integration gate indicated.

We have implernented two techniques to caprure more of the information-containing signal in order to increase the sensitivity of the method. In the furst technique the strongest part of the waveform from the detector, approximately the first $100 \mu \mathrm{sec}$, is repetitively acquired and averaged using a digitizing oscilloscope and transferred to a computer, where it is rectified and integrated to give the relative PAS signal. Figure ib shows a typical amplified voltage waveform after the rectification step, along with the interval over which integration is peiformed. Since the HP54111D oscilloscope which we use can be pretriggered, we are also able to integrate the time region before the laser pulse reaches the sample and subtract this from the measured signal. This enables us to eliminate lowfrequency noise and dc offset.

The second technique uses a simple analog processing module which converts the ac voltage waveforms from the detector to their root mean square (rms) values in real time, producing essentially rectified envelope functions of the incident waveform. The major component in the circuit is a MAXIM AD636 RMS to DC converter chip. Figure lc shows a waveform before and after conversion. The resultant waveform contains the relevant 
intensity information from the original waveform and can be completely integrated using a gated integrator, thus using much more of the available acoustic energy than the gated integrator only technique, but requiring much less signal processing time than the digital oscilloscope technique.

Suspended particulate matter in the sample solutions can be a severe problem when obtaining spectra of very dilute solutions. Acoustic signal from interaction of the laser with particulates completely overwhelms the signal from interaction with the anilyte. Filtration of all samples with $0.2 \mu \mathrm{m}$ syringe filters prior to loading into the sample cells serves to greatly reduce the particulate problem but does not completely eliminate it. Direct light scartering from remaining particulates and from sample cell surfaces can be reduced by coupling the sample cell to the piezoelectric detector through a glass or quartz microscope slide.

In all of the techniques as implemented by us, real-time data processing routines are included in the scanning software to reduce or eliminate the potential spectral noise introduced by particulate matter in the sample solutions. The central idea is to have the software exclude data from the average used to produce a point in the spectrum if the data is outside of certain limits from the running average. We also routinely store and plot as error bars the statistical information at the $95 \%$ confindence interval on each data point.

In parallel with the PAS experimental apparatus, we have implemented photothermal lensing detection of low level absorption in solution. The dual-beam, twolaser technique which we use incorporates pulsed dye laser excitation, a HeNe probe laser and photodiode detection with low-noise amplification and high-pass froquency filtering. The theory and experimental design have been developed in some detail by others. 5,6 Radiationless relaration of the analyte following laser pumping of an electronic transition releases heat into the sample solution in the region through which the pump laser passes. The refractive index gradient resulting from the temperature-dependent index of refraction produces a negative lens in aqueous solution at room temperature. This transient lens defocuses a the HeNe probe beam passing through it, and the defocusing is detected as a transient decrease in intensity of the center of the HeNe beam as detected in the far field by a photodiode behind a pinhole. The perturbation of the probe laser intensity at the detector is long-lived, having a lifetime of about $20 \mathrm{msec}$. The photodiode signal after two different high-pass filtering schemes is shown in Figure 2. A gated integrator is used to measure the intensity of the high-frequency front edge of the lens formation in time after the pump pulse. Although high-pass filtering prevents us from using all of this potential signal, the drastic lowering of the noise level which it produces gives a net improvement of signal-tonoise. We have also experimented with differential thermal lens techniques ${ }^{7}$ to eliminate water absorption and other sources of background signal from the spectra. 

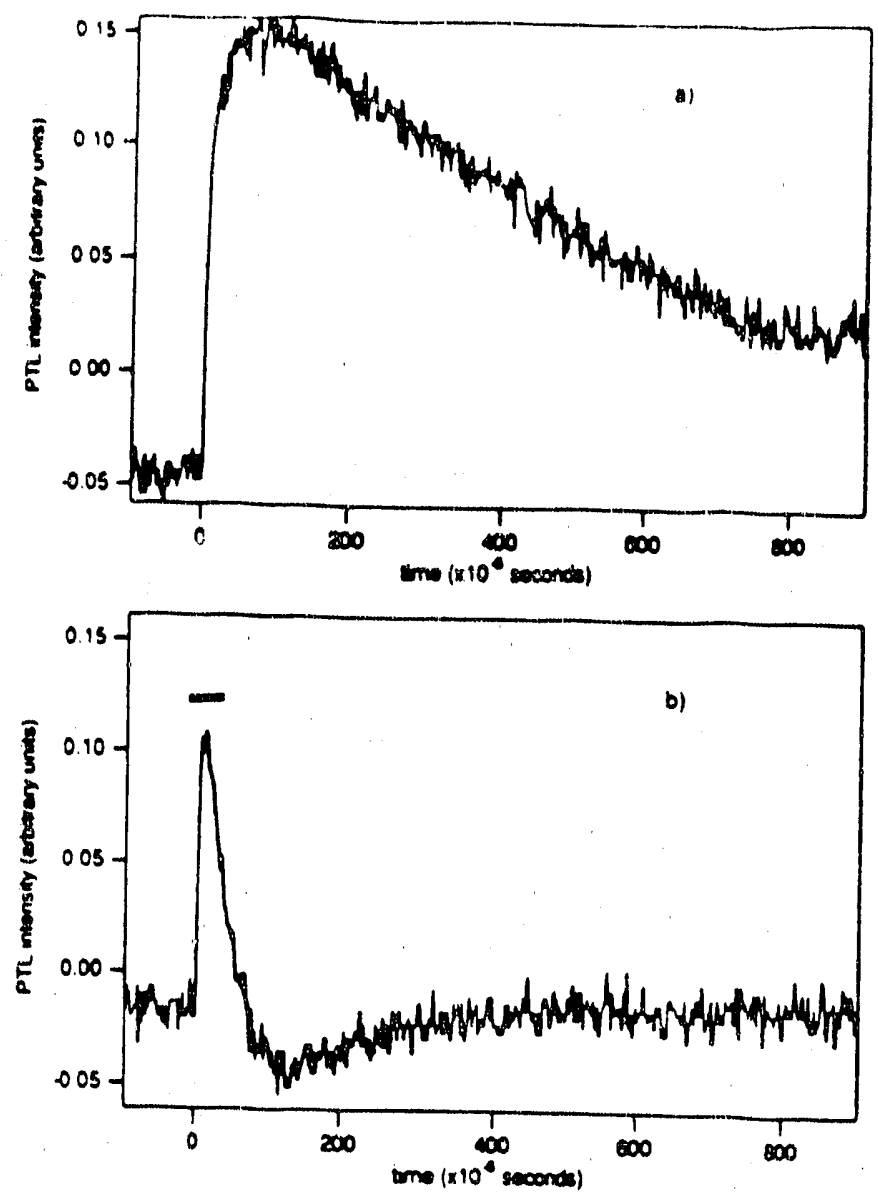

Figure 2. Thermal lensing signals with two different high-pass filter settings: a) $100 \mathrm{~Hz}$ high-pass; b) $3 \mathrm{kHz}$ high-pass. Actual spectra were recorded using the $3 \mathrm{kHz}$ filter and the integration gate indicated by $a$ bar in the spectrum.

\section{RESULTS AND DISCUSSION}

Our assessment of the relative advantages of the three PAS signal processing techniques discussed above is presented in Table I.

Table I: Comparison of rechniques for processing PAS signals.

\begin{tabular}{|c|c|c|}
\hline Technique & Advantages & Disadvantages \\
\hline Boxcar & $\begin{array}{l}\text { Fast } \\
\text { Relatively inexpensive } \\
\text { Avoids later time interferences }\end{array}$ & $\begin{array}{l}\text { Uses only small part of the acoustic } \\
\text { waveform } \\
\text { Arbitrary selection of excursion to } \\
\text { use }\end{array}$ \\
\hline Digital Scope & $\begin{array}{l}\text { Uses most of detected acoustic } \\
\text { energy } \\
\text { Adds capability for waveform } \\
\text { analysis } \\
\text { Can exclude noisier time windows }\end{array}$ & $\begin{array}{l}\text { Relatively equipment-intensive, } \\
\text { expensive } \\
\text { More computer overhead, slower on } \\
\text { current systems } \\
\text { Subject to interferences }\end{array}$ \\
\hline $\begin{array}{l}\text { Analog } \\
\text { Processor }\end{array}$ & $\begin{array}{l}\text { Fast: real-time processing of each } \\
\text { laser shot } \\
\text { Relatively inexpensive } \\
\text { Uses most of detected acoustic } \\
\text { energy } \\
\text { Minimal computer overthead }\end{array}$ & $\begin{array}{l}\text { Subject to interferences from } \\
\text { windows etc -. can't be gated } \\
\text { out easily } \\
\text { Waveform deconvolution not } \\
\text { possible }\end{array}$ \\
\hline
\end{tabular}


Typical spectra of $10 \mu \mathrm{M} \mathrm{Nd} d^{3+}$ in $0.1 \mathrm{~N} \mathrm{HCl}$ using the three signal processing methods discussed above are shown in Figure 3. Error bars represent two standard deviations of the set of data which were averaged to give each of the points on the spectra. The signal-to-noise ratios in the three spectra are comparable, though some improvement can be noted in both the digital oscilloscope spectrum (Fig 3b) and the analog rectification spectrum (Fig 3c) over the the gated integrator only spectrum (Fig 3a).

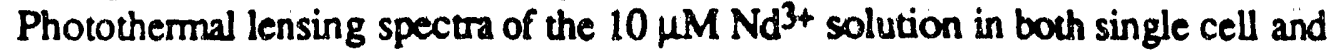
background subtraction mode are shown in Figure 4 for comparison with the PAS results. The $\mathrm{S} / \mathrm{N}$ on the $\mathrm{Nd}^{3+}$ peak at $522 \mathrm{~nm}$ is comparable to that seen in the PAS spectra. The rising edge between 505 and $515 \mathrm{~nm}$ is due to increasing water absorption. We seem to be able to achieve more consistent water background signals with PTL than with PAS. The background subtraction technique appears to work well, but it increases the difficulty of the experiment because one must carefully balance the power of the pump laser and the focusing parameters of both lasers in the two cells.

A PAS spectrum of $2 \mu \mathrm{M}$ Pu(IV) in $3 \mathrm{M}$ carbonate solution (pH 12.1) is shown in Figure 5. This spectrum was obtained using the digital oscilloscope signal processing scheme. Note that the error bars produced by statistical analysis of the data significantly enhance ones ability to differentiate between real spectroscopic features and noise. We hope to have spectra of Pu(TV) in the nanomolar range in the near future.
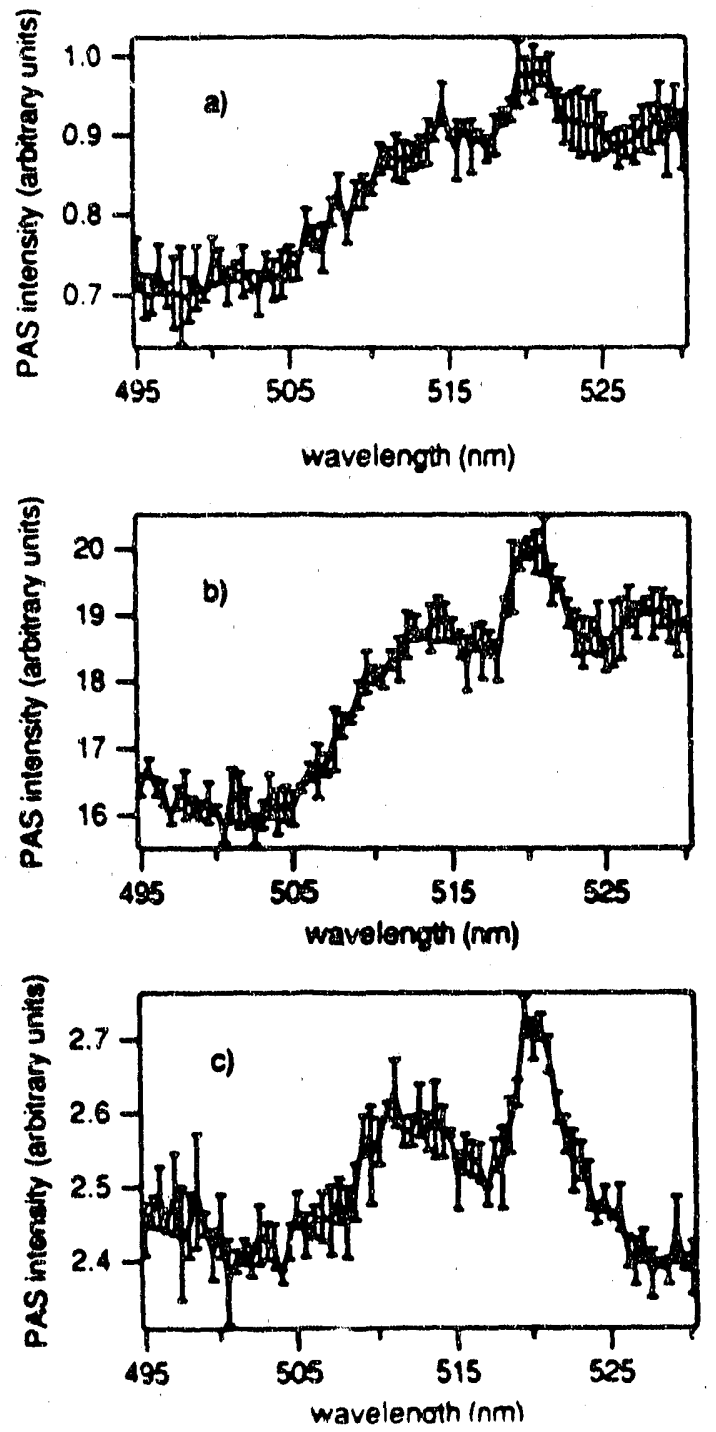

Figure 3. Photoacoustic spectra of $10 \mu \mathrm{M} \mathrm{Nd}^{3+}$ in $0.1 \mathrm{~N} \mathrm{HCl}$ solution taken using three different signal processing techniques: a) boxcar integration with gate shown in Fig. 1a; b) digital oscilloscope waveform collection followed by computer rectification and integration over the time interval indicated in Fig $1 \mathrm{~b} ; \mathrm{c}$ ) analog rectification followed by integration over the time interval indicated in Fig. Ic. The optical density of $\mathrm{Nd}^{3+}$ in $0.1 \mathrm{~N} \mathrm{HCl}$ is $4.0 / \mathrm{cm}$ at $522 \mathrm{~nm}$. 

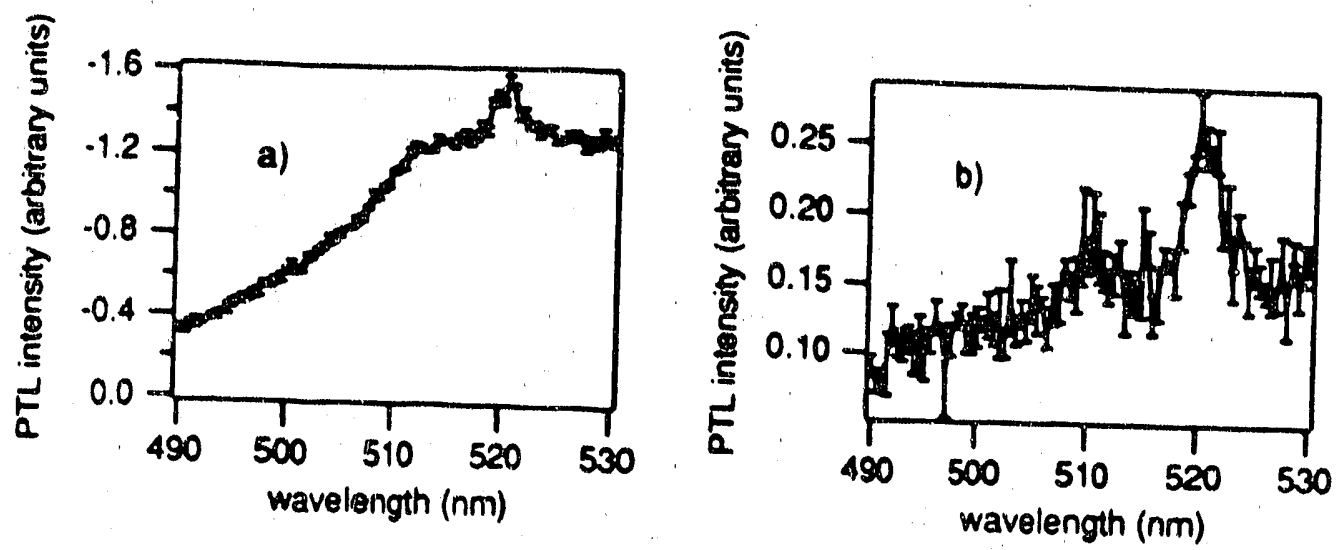

Figure 4. Photothermal lensing spectra of $10 \mu \mathrm{M} \mathrm{Nd} d^{3}+$ in $0.1 \mathrm{~N} \mathrm{HCl}$ solution: a) single cell spectrum; b) dual cell, dual beam spectrum with $0.1 \mathrm{~N} \mathrm{HCl}$ as the reference solution.

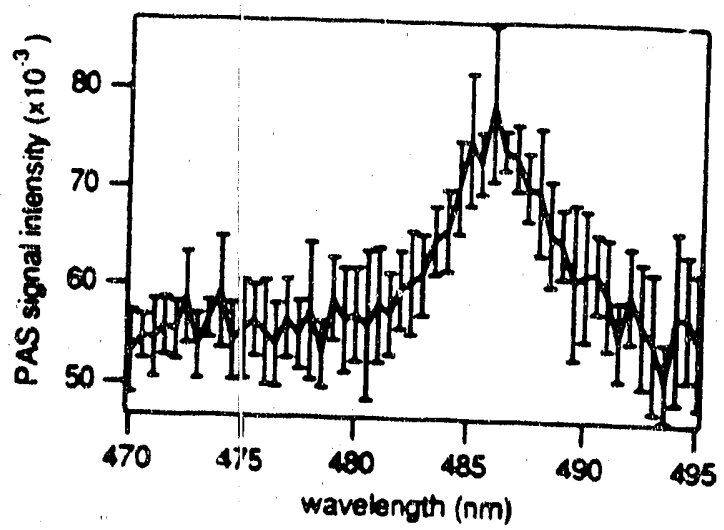

Figure 5. Photoacoustic spectrum of $2 \mu M \mathrm{Pu}(\mathrm{IV})$ in 3M carbonate solution ( $\mathrm{pH}=12.1$ ) acquired using digital oscilloscope.

We do not claim that our PAS spectra represent improvements in sensitivity over that achieved by other groups, and in fact they fall somewhat short of the best sensitivity claims of which we are aware. ${ }^{8}$ Our emphasis is on exploration of alternative signal processing schemes which may lead to overall improved sensitivity when coupled with the advances made by other groups in areas such as sample cell-detector coupling and detector design.

The PAS signal processing schemes which use more of the waveform show potential for improving sensitivity without adding complexity to the experiment. Some aspects need further work, however. The analog reutification scheme seems to have introduced an inconsistency in the baseline observed beneath known spectra (see Fig. 3c). This effect probably originates from interferences within the waveform at later time between the signal from the analyte and $\mathrm{H}_{2} \mathrm{O}$ and the signal from other sources (e.g. electrostriction, window absorption, light scatter, if pickup.) We have begun exploring ways of getting rid of the effect of these interferences by taking differences between waveforms produced ai different wavelengths prior to subsequent manipulation. Interestingly, the resultant difference waveform appears very similar to waveforms 
published by Fisher, Fasano and Nogar9 in that they settle to a single dominant frequency component at long times. Fisher et al. suggested extracting analytically useful signal intensities from waveforms by Fourier transforming the PAS waveform and measuring only this resonant frequency component. The results are interesting because they indicate that interferences which reduce apparent signal intensity are definitely more pronounced at later tirnes, and they also indicate that differences between waveforms taken at different wavelengths can be used to produce spectra in which systematic errors such as the baseline slope in Fig $3 c$ are largely eliminated. Considerable work needs to be done before we know whether this idea may reasonable be incorporated into routine acquisition of spectra.

1 W. Schrepp, R. Stumpe, J. I. Kim and H. Walther, Appl. Phys. B 32, 207 (1983).

R. Stumpe, J. I. Kim, W. Schrepp and H. Walther, Appl. Phys. B 34, 203 (1984).

S. C. Rutan and S. D. Brown, Anal. Chim. Acta 158, 113 (1984).

M. Eiswirth, J. I. Kim and C. Lierse, Radiochimica Acta 38, 197 (1985).

J. V. Beizz, K. L. Bowers, M. M. Doxtader, V. A. Maroni and D. T. Reed, Radiochimica Acta, 44/45, 87 (1988).

R. A. Torres, C. E. A. Palmer, P. A. Baisden, R. E. Russo and R. J. Silva, Anal. Chem. 62, 298 (1990).

J. V. Beiu., M. M. Doxtader, V. A. Maroni, S. Okajima, and D. T. Reed, Rev. Sci. Instrum. 61, 1396 (1990).

2 T. Berthoud, P. Mauchien, N. Omenetto and G. Rossi, Anal. Chim. Acta 153, 265 (1983).

G. Bidoglio, G. Tanet, P. Cavalli and N. Omenetoo, Inorg. Chim. Acta 140, 293 (1987).

C. Moulin, N. Delorme, T. Berthoud and P. Mauchien, Radiochimica Acla W/45, 103 (1988).

I. Grenthe, G. Bidoglio and N. Omenetuo, Inorg. Chern. 28, 71 (1989).

3 A. C. Tam, Rev. Mod. Phys. 58, 381 (1986).

C. K. N. Patel and A. C. Tam. Rev. Mod. Phys. 53, 517 (1981).

4 H. M. Lai and K. Young, J. Acoust. Soc. Am 72, 2000 (1982).

${ }^{5}$ H. L. Fang and R. L. Swofford, in Ulirasensitive Laser Spectroscopy (Academic Press, New York, 1983) pp. 175-232.

6 T. Berthoud, N. Delorme and P. Mauchien, Anal, Chem 57, 1216 (1985).

7 S. R. Erskine and D. R. Bobbitt, Appl. Spec. 43, 668 (1989).

8 J. V. Beitz, M. M. Doxtader, V. A. Maroni, S. Okajima and D. T. Reed, Rev. Sci. Instrum. 61, 1396 (1990).

9 M. R. Fisher, D. M. Fasano and N. S. Nogar, Appl. Spectros. 36, 125 (1982). 

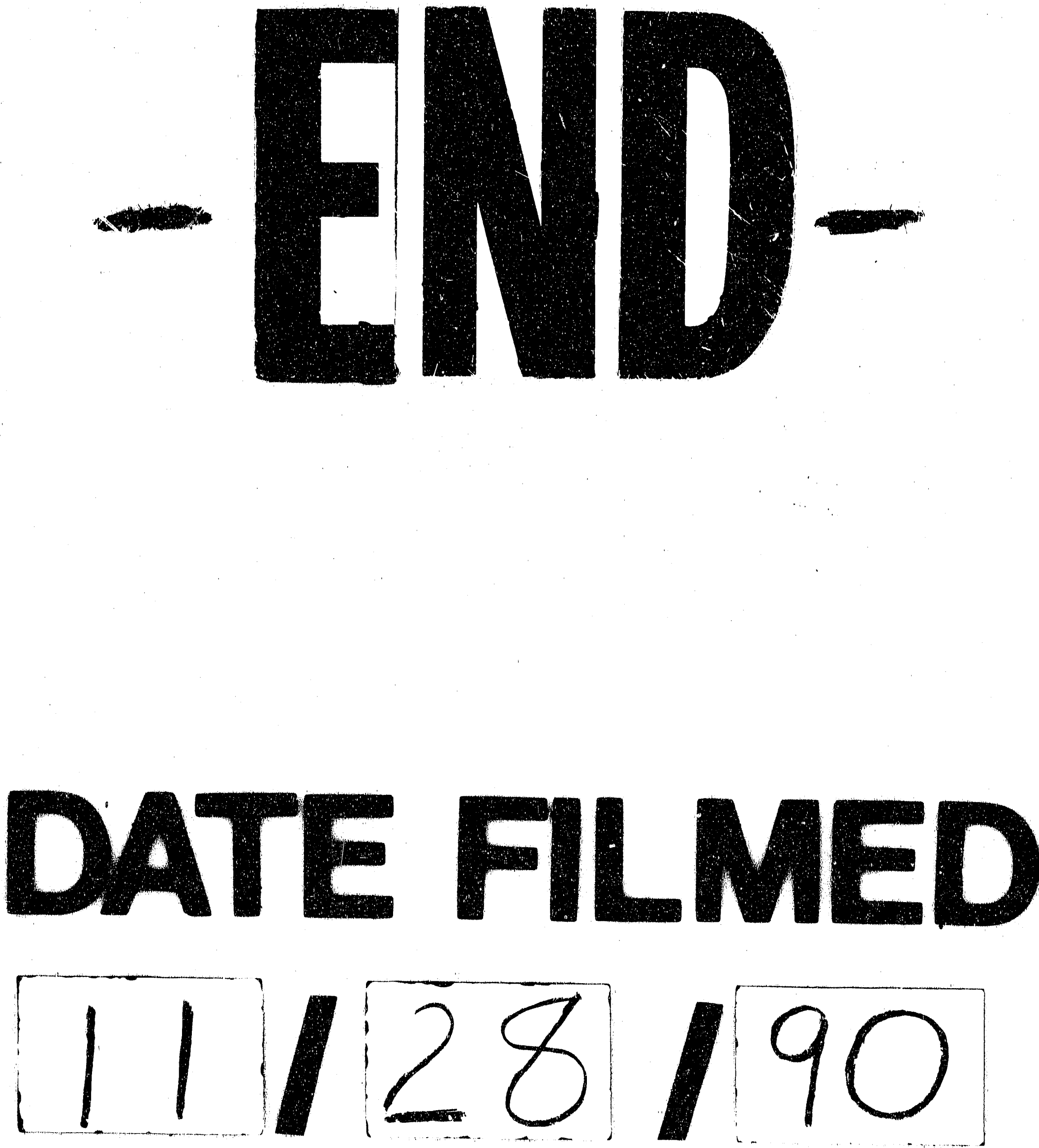
